\title{
Recovering Estimates of Fluid Flow from Image Sequence Data
}

\author{
Richard P. Wildes, Michael J. Amabile, Ann-Marie Lanzillotto, and Tzong-Shyng Leu ${ }^{1}$ \\ Sarnoff Corporation, CN5300, Princeton, New Jersey 08543
}

Received December 2, 1999; accepted August 2, 2000

\begin{abstract}
This paper presents an approach to measuring fluid flow from image sequences. The approach centers around a motion-recovery algorithm that is based on principles from fluid mechanics: The algorithm is constrained so that recovered flows observe conservation of mass as well as physically motivated boundary conditions. Empirical results from application of the algorithm to transmittance imagery of fluid flows, where the fluids contained a contrast medium, are presented. In these experiments, the algorithm recovered accurate and precise estimates of the flow. The significance of this work is twofold. First, from a theoretical point of view it is shown how information derived from the physical behavior of fluids can be used to motivate a flow-recovery algorithm. Second, from an applications point of view the developed algorithm can be used to augment the tools that are available for the measurement of fluid dynamics; other imaged flows that observe compatible constraints might benefit in a similar fashion. (c) 2000 Academic Press
\end{abstract}

\section{INTRODUCTION}

\subsection{Motivation}

Physical modeling plays an important role in computer vision. Constraints derived from physical considerations are often used to provide the basis for well-motivated algorithms. This close tie between image interpretation and physical modeling suggests that measurement problems in the physical sciences might be a fruitful source of research issues for computer vision. A particular domain where this methodology is likely to be of value is the measurement of fluid flow from image sequences. Here, applicable physical constraints come naturally from fluid mechanics. For example, differential flow constraints can arise from the continuity equations yielded by conservation of mass and momentum. Similarly, realistic boundary conditions can be derived from physical considerations. Significantly,

\footnotetext{
${ }^{1}$ The research that is described in this paper was funded by DARPA/ETO under Contract DABT63-95-C-0057.
} 
it is standard practice in experimental fluid mechanics to seed flows with tracers for the sake of visualization. This practice yields imagery with the patterned contrast (i.e., texture) necessary to drive standard computer vision motion-analysis algorithms. Owing to their physical underpinning, algorithms that are founded on these principles should exhibit greater accuracy and precision than those based more simply on the matching of image intensity patterns.

Motivated by these observations the current paper presents an algorithm, based in the physics of fluid mechanics, for the recovery of fluid flow from image sequences. The algorithm is particularly targeted to the recovery of two-dimensionally imaged motion of three-dimensional media. Significantly, such two-dimensional information can be of importance in and of itself, even though the actual three-dimensional motion is not made explicit. As examples, in fluid mechanics, two-dimensional motion allows comparisons between empirical data and theoretical models (Lanzillotto et al. [1]); in medical image analysis, two-dimensional motion can be used to monitor blood flow in a meaningful fashion (Amini [2], Nogawa et al. [3]); in meteorology, two-dimensional motion in atmospheric image sequences can be used to support large-scale pattern analysis (Cohen and Herlin [4], Larsen et al. [5], Memin and Perez [6]). The immediate application of the developed algorithm is to the experimental study of fluidic devices. More generally, however, the developed algorithm should be applicable to a variety of flow-recovery problems where the kinematics of imaged motion is related to the continuum mechanics of the imaged media. In particular, certain types of (i) medical transmittance imaging (e.g., X-ray time series of biological tissue (Webb [7])) and (ii) visible reflectance imaging (e.g., uncalibrated image sequences depicting dilation and/or angular rotation (Del Bimbo et al. [8])) might be well suited to the described algorithm.

\subsection{Related Research}

The recovery of optical flow, i.e., the apparent motion of image intensity patterns, has been the subject of a great deal of research (Aggarwal and Nandhakumar [9], Beauchemin and Barron [10]). Typically, optical flow algorithms are based on the brightness constancy constraint (Horn [11]). Essentially, this dictates that the algorithms establish a mapping between two images based directly on the similarities of the image intensities. Various phenomenological extensions to this constraint have been proposed to make it less restrictive (e.g., Cornelius and Kanade [12], Negahdaripour and Yu [13]). Other extensions have been based on the analysis of the imaging of three-dimensional objects under perspective projection (Nagel [14]). For many applications, algorithms based on brightness constancy or the cited extensions have proven to be of value. However, these constraints fail to adequately capture the nature of fluid flow and therefore are less clearly applicable to that problem domain.

For the matters at hand, a natural constraint arises in the flow continuity equation as derived from the principle of conservation of mass. This constraint dictates temporal image transformations that are consistent with fluid flow behavior. Schunck [15] first applied the conservation of mass constraint to the analysis of imaged motion. This work suggested the possibility of using the conservation of mass constraint to model the imaged motion of rotating and perspectively distorted objects; however, it did not propose a method to solve for corresponding flows given image irradiance data. Fitzpatrick [16] appears to have presented the first specific solution method, with the additional constraint that the flow 
is irrotational. While this work did not present an implemented algorithm, it did present an analytic example. Subsequently, Fitzpatrick and Pedersen [17] documented the first working algorithm with results on natural imagery. This algorithm incorporated not only the conservation of mass and irrotational flow constraints, but also the use of physically realistic boundary conditions. In conjunction with this work, Fitzpatrick [18] presented a theoretical analysis that showed the relationships between a three-dimensional medium that obeys the conservation of mass and its corresponding two-dimensional transmittance image. In particular, this work justified the application of the conservation of mass continuity equation in the image domain even for the case of discontinuous media, via consideration of the blurring that is inherent in the imaging device. This work is of key importance for the research that is reported in the current paper, where the conservation of mass is applied to the analysis of two-dimensional transmittance imagery of three-dimensional discontinuous fluids (i.e., fluids containing a contrast medium).

Song and Leahy [19] derived the conservation of mass continuity equation in their presentation; however, they ultimately combined it with an incompressibility constraint to yield the continuity equation for incompressible media. In implementation, this work reported an algorithm that recovered three-dimensional flow from corresponding image sequence data while enforcing a small divergence constraint. In this case, the small divergence constraint was justified by the target application domain (analysis of cine computed tomograms of the heart). Amini [2] also reported use of the conservation of mass constraint in conjunction with incompressibility in an algorithm with application to the analysis of blood flow. In this case it was shown that if incompressible flow along one of its three spatial dimensions is equal to zero and the medium is described by a two-dimensional distribution in the other spatial coordinates, then the continuity equation reduces to the brightness constancy constraint. Yet another approach to incorporating the conservation of mass constraint has been to approximate its solution via application of least-squares methods to a truncated Taylor series expansion of the continuity equation (Del Bimbo et al. [20]).

Interestingly, not all work from the computer vision and image processing communities that has been concerned with recovering fluid flow from imagery has made direct use of conservation of mass inspired continuity constraints (Jähne and Waas [21], Maas et al. [22], Cohen and Herlin [4], Larsen et al. [5], Memin and Perez [6]). Similarly, standard techniques developed in experimental fluid mechanics for recovering fluid flow from imagery have not made direct use of conservation of mass inspired continuity constraints. Instead, they have relied on image correlation techniques (occasionally with extensions) or simple particle trackers applied to images of fluids containing a contrast medium (Adrian [23], Lourenco et al. [24], Willert and Gharib [25], Zhong et al. [26]). Finally, an alternative approach developed especially for fluid dynamics phase portrait visualization relies on recovery of the local tangent field to images depicting streamlines of the underlying flow (Ford et al. [27], Ford and Strickland [28]).

The current work contributes to previous research mainly as follows. The conservation of mass flow continuity equation is used in a variational formulation of a novel algorithm for the recovery of fluid flow from image sequences. This algorithm has been implemented and successfully evaluated with both synthetic and natural imagery of microfluidics. While previous research has made use of the conservation mass constraint in the development of algorithms for image motion analysis, it appears that the current work is the first that does not require either irrotational or small-divergence flow. Indeed, there is a cause and effect relationship between these various formulations and the flows that the algorithms recover. 
Ultimately, the appropriateness of one or another approach depends on the application domain that is under consideration. Preliminary versions of the research that is reported in the current paper have appeared previously (Wildes et al. [29, 30]).

\subsection{Outline of Paper}

The main body of this paper unfolds in four sections. The first section has served to motivate physics-based recovery of fluid flow from image data. Section 2 describes a technical approach to this matter. In Section 3, experimental results of testing the approach in the face of fluid flow imagery are provided. Section 4 provides a summary. Following the main sections an appendix provides additional details on image formation.

\section{TECHNICAL APPROACH}

\subsection{Algorithm Derivation}

Let $E(x, y, t)$ be an image, a function of spatial coordinates, $(x, y)$, and time, $t$. Suppose that this image depicts a fluid flow in such a way that the essential physical characteristics of the flow are captured. In particular, suppose that the imaged intensities observe the conservation of mass just as does the fluid density. For example, in the Appendix to this paper it is shown that the two-dimensional transmittance image of a three-dimensional fluid flow that respects conservation of mass in three dimensions is a two-dimensional flow that respects the conservation of mass in two dimensions. This is true provided that there is no material loss due to normal flow at the boundaries of the flow. In this case the twodimensional (imaged) flow is the density-weighted average of the three-dimensional flow, taken along imaging rays. Correspondingly, application of the conservation of mass flow continuity equation to a temporally varying image yields

$$
E_{x} u+E_{y} v+E u_{x}+E v_{y}+E_{t}=0
$$

or more compactly

$$
\nabla \cdot(E \mathbf{v})+E_{t}=0
$$

where $\mathbf{v}=(u(x, y, t), v(x, y, t))$ is the imaged flow, $\nabla \equiv\left(\frac{\partial}{\partial x}, \frac{\partial}{\partial y}\right)$ is the spatial gradient operator, and subscripts denote partial differentiation (Streeter [31]). In essence, this constraint states that flow through the boundary of a region must be balanced by accumulation in the region. The continuity equation (1) will be taken as the fundamental constraint for relating image intensity measurements to fluid flow. In this regard, it can be contrasted with the brightness constancy constraint

$$
E_{x} u+E_{y} v+E_{t}=0,
$$

the standard constraint for optical flow algorithms. This constraint more simply insists on identity of intensities between corresponding image elements across time. Notice that the brightness constancy assumption is too restrictive a model for fluid flow, where material particles may well deform (in a conservative fashion), whereas the conservation of mass 
continuity equation accurately captures such phenomena. In practice, to allow for imperfect data, strict enforcement of the continuity constraint is replaced with minimization of

$$
c_{c}=\left(\nabla \cdot(E \mathbf{v})+E_{t}\right)^{2},
$$

with respect to $\mathbf{v}$ over an image domain of interest.

To operate successfully with imperfect data, it is useful not only to avoid strict adherence to the continuity constraint, but also to impose additional constraints on the recovered flow that are intended to ameliorate the effects of noise. A useful constraint in this regard is to encourage smoothness of the recovered flow. This notion can be captured by minimizing the spatial variation of the flow; e.g.,

$$
c_{s}=u_{x}^{2}+u_{y}^{2}+v_{x}^{2}+v_{y}^{2} .
$$

Another way to look at this constraint is in terms of regularization theory, where constraints of this form are taken to stabilize the solution (Tikhonov and Arsenin [32]). Without imposition of this constraint, it was found that the imagery of interest was too noisy to yield coherent flow fields.

Following the methodology of Horn and Schunck [33], the measures of continuity (3) and smoothness (4) can be combined and evaluated over a domain of interest to yield a problem of the form

$$
\min \iint\left(\lambda c_{c}+c_{s}\right) d x d y
$$

with $\lambda$ being a weighting parameter that trades off adherence to the continuity constraint and smoothness of flow. Minimization of this integrated constraint equation with respect to flow parameters $(u, v)$ is a problem in the calculus of variations (Courant and Hilbert [34]). In particular, the integrand of Eq. (5) has the form $F\left(x, y, u, v, u_{x}, u_{y}, v_{x}, v_{y}\right)$. The corresponding Euler-Lagrange equations, which provide necessary conditions for a minimum, are

$$
\begin{aligned}
& F_{u}-\frac{\partial}{\partial x} F_{u_{x}}-\frac{\partial}{\partial y} F_{u_{y}}=0 \\
& F_{v}-\frac{\partial}{\partial x} F_{v_{x}}-\frac{\partial}{\partial y} F_{v_{y}}=0 .
\end{aligned}
$$

When these equations are evaluated in terms of the integrated constraint equation (5), a pair of partial differential equations (PDEs) results,

$$
\begin{aligned}
& \nabla^{2} u=-\lambda\left(E_{t x}+E_{x x} u+E_{y x} v+2 E_{x} u_{x}+E_{y} v_{x}+E_{x} v_{y}+E v_{y x}+E u_{x x}\right) E \\
& \nabla^{2} v=-\lambda\left(E_{t y}+E_{x y} u+E_{y y} v+E_{y} u_{x}+E_{x} u_{y}+2 E_{y} v_{y}+E u_{x y}+E v_{y y}\right) E,
\end{aligned}
$$

where $\nabla^{2} \equiv\left(\frac{\partial^{2}}{\partial x^{2}}+\frac{\partial^{2}}{\partial y^{2}}\right)$ is the Laplacian operator. These imaged fluid flow equations relate image intensity measurements to permissible flow field components as constrained by conservation of mass and smoothness of flow.

In order for a variational problem, such as minimization of the integrated constraint equation (5) to be well posed, it is necessary to enforce appropriate boundary conditions. 
Physical considerations can serve to provide the needed constraints. For fluidic systems, a basic constraint is that the fluids must flow within their containing channels (essentially, impenetrability of matter). This can be expressed by disallowing normal flow along the boundaries of the domain in which the fluid lies. In the Appendix to this paper, it is shown that operation of this constraint in a three-dimensional specimen implies a corresponding two-dimensional boundary condition in its transmittance image; i.e.,

$$
[\mathbf{n} \cdot \mathbf{v}]_{\partial \omega}=0,
$$

where $\mathbf{n}$ specifies the normals to the two-dimensional imaged boundary, $\partial \omega$, of the threedimensional specimen. Additionally, if the (imaged) channel boundaries do not completely enclose the domain of interest (e.g., the apparent end of a channel as it runs off the end of the image), then natural boundary conditions (Neuman boundary conditions) are enforced at such points. Applying natural boundary conditions at channel endings is appropriate for the steady state flows that are the subject of this paper's experimental evaluation; however, alternative constraints might be more applicable in other scenarios.

A numerical solution to the imaged fluid flow equations, (6), can be found via discretization. Application of finite differences (Dahlquist and Björck [35]) to these equations yields

$$
\begin{aligned}
4\left(\bar{u}_{i, j}-u_{i, j}\right)= & -\lambda\left[E_{t x}+E_{x x} u_{i, j}+E_{y x} v_{i, j}+2 E_{x} \Delta_{x} u_{i, j}+E_{y} \Delta_{x} v_{i, j}+E_{x} \Delta_{y} v_{i, j}\right. \\
& \left.+E \Delta_{y x} v_{i, j}+2 E\left(\bar{u}_{i, j}^{x}-u_{i, j}\right)\right] E \\
4\left(\bar{v}_{i, j}-v_{i, j}\right)= & -\lambda\left[E_{t y}+E_{x y} u_{i, j}+E_{y y} v_{i, j}+E_{y} \Delta_{x} u_{i, j}+E_{x} \Delta_{y} u_{i, j}+2 E_{y} \Delta_{y} v_{i, j}\right. \\
& \left.+E \Delta_{x y} u_{i, j}+2 E\left(\bar{v}_{i, j}^{y}-v_{i, j}\right)\right] E,
\end{aligned}
$$

where $i, j$ is the index of image position, $\Delta_{x} u_{i, j}=\left(u_{i+1, j}-u_{i-1, j}\right) / 2$ is the central difference, $\Delta_{x y} u_{i, j}=\left(u_{i+1, j+1}-u_{i+1, j-1}-u_{i-1, j+1}+u_{i-1, j-1}\right) / 4$ is the mixed difference, $\bar{u}_{i, j}^{x}=\left(u_{i+1, j}+u_{i-1, j}\right) / 2$ and $\bar{u}_{i, j}=\left(u_{i+1, j}+u_{i-1, j}+u_{i, j+1}+u_{i, j-1}\right) / 4$ are averaging operators, etc. These equations can be written more compactly by letting $\mathcal{I}$ be the identity matrix,

$$
\begin{aligned}
\mathcal{A} & =-E\left(\begin{array}{cc}
E_{x x}-E & E_{y x} \\
E_{x y} & E_{y y}-E
\end{array}\right), \\
\mathbf{b}(\mathbf{v}) & =E\left(\begin{array}{c}
E_{t x}+2 E_{x} \Delta_{x} u_{i, j}+E_{y} \Delta_{x} v_{i, j}+E_{x} \Delta_{y} v_{i, j}+E \Delta_{y x} v_{i, j}+E \bar{u}_{i, j}^{x} \\
E_{t y}+E_{y} \Delta_{x} u_{i, j}+E_{x} \Delta_{y} u_{i, j}+2 E_{y} \Delta_{y} v_{i, j}+E \Delta_{x y} u_{i, j}+E \bar{v}_{i, j}^{y}
\end{array}\right),
\end{aligned}
$$

$\mathbf{v}=(u, v)^{T}$ and $\overline{\mathbf{v}}=(\bar{u}, \bar{v})^{T}$, to yield

$$
(\mathcal{I}+\lambda \mathcal{A}) \mathbf{v}=\overline{\mathbf{v}}+\lambda \mathbf{b}
$$

Various numerical methods exist that could solve the previous system for $\mathbf{v}$ (see, e.g., Dahlquist and Björck [35]). For example, in theory it would be possible to apply a method such as Gauss-Jordan elimination directly to this discrete system. However, due to the sparseness and size of the resulting matrix (i.e., the number of rows and columns equals two times the number of image pixels), this is a needlessly costly approach. Instead, a method that has been successful in the current context is algorithmic time discretization. 
In particular, application of the inverse of $\mathcal{I}+\lambda \mathcal{A}$ to both sides of the previous equation yields an iterative solution for $u$ and $v$,

$$
\mathbf{v}^{n+1}=(\mathcal{I}+\lambda \mathcal{A})^{\dagger}\left(\overline{\mathbf{v}}^{n}+\lambda \mathbf{b}\left(\mathbf{v}^{n}\right)\right)
$$

where $\mathbf{v}^{n+1}$ is the value of $\mathbf{v}=(u, v)^{\top}$ computed at iteration $n+1$ from the value $\mathbf{v}^{n}$ computed at iteration $n$ and $\dagger$ denotes the matrix inverse. Intuitively, these equations can be taken as providing updated values for $(u, v)$ based on local averages of the flow field as corrected by deviation from conservation of mass dictated continuity.

\subsection{Algorithm Instantiation}

The iterative solution for flow field components (8) has been embodied in a Gauss-Seidel relaxation algorithm; i.e., new values of $\mathbf{v}$ are entered into the computation as soon as they are calculated rather than at the completion of an entire iteration cycle as done in Jacobi relaxation (Dahlquist and Björck [35]). This algorithm accepts a pair of images as well as a region of interest map and recovers a corresponding flow field. The region of interest map is a binary map that demarks the areas over which flow is to be calculated. The algorithm first solves for the flow along the channel boundary of the region of interest while enforcing the null normal flow constraint (7). This constraint is practically instantiated in the following fashion. Along each channel wall the imaged flow equations (6) are solved as restricted to one dimension. This yields a single one-dimensional flow equation, with the direction constrained to be tangential to the channel wall. To ensure that this problem also is properly posed, natural boundary conditions (i.e., Neumann boundary conditions) are enforced at the ends of the channel. These are implemented by introducing a new point at each end of the channel boundary and copying over the one-dimensional solution at the neighboring interior point at each iteration. Subsequently, the one-dimensional solution(s) are used as the boundary conditions as the algorithm solves on the domain interior. During this process, natural boundary conditions are enforced at loci where the channel walls do not completely enclose the domain of interest (e.g., the apparent end of a channel as it runs off the end of the image). The initial values for $u$ and $v$ are taken as zero everywhere.

This relaxation algorithm has been embedded in a hierarchical coarse-to-fine refinement control structure to support extended spatial capture, i.e., recovery of multipixel motions, and computational efficiency (Bergen et al. [36]). Operation begins by computing a series of low-resolution versions of the input images (via successive one-octave low-pass filtering and factor of 2 subsampling). The coarsest resolution is then used to recover an initial flow estimate which is refined via reference to successively finer resolution images, finishing with the original images. In this regard, it is important to note that the conservation of mass flow continuity equation is applied at each iteration of the estimation process. It is a heuristic assumption that the constraint is thereby enforced on the overall displacement that results from the concatenation of the individual estimates. Nevertheless, empirical results (as described in the next section of this paper) support this methodology.

In the experiments reported in this paper coarse-to-fine processing proceeded up to five levels of resolution reduction. Iterations of the flow field solution (8) were calculated to allow the most distant pixels at the coarsest resolution to broadcast their intensity values to one another. For example, starting with a $512 \times 512$ image reduced by 5 levels to yield a $16 \times 16$ image leads to $\lceil 16 \sqrt{2}\rceil=23$ iterations being calculated at each resolution level. 
Values for $\lambda$ were selected empirically; specific values are documented in the context of the experiments described in Section 3. This entire scheme has been implemented in the $\mathrm{C}$ programming language. As currently installed, it executes from the UNIX command line on Sun SPARCstations and as an Explorer module on Silicon Graphics workstations.

\section{EXPERIMENTAL EVALUATION}

It is hypothesized that the developed flow-recovery algorithm is widely applicable to the measurement of fluid flow from imagery at a wide range of spatial scales. The current application domain for the algorithm is microfluidics (Lanzillotto et al. [1]). Of particular interest is employing the algorithm in conjunction with microradiography (Cosslett et al. [37]) to enable the noninvasive assessment of microelectromechanical systems (MEMS). Therefore, the reported experiments concentrate on microscale X-ray imagery. Further, all of the considered flows have small Reynolds numbers, approximately equal to one. (Reynolds number is the ratio of inertial to viscous forces; smaller values are associated with higher stability of laminar (as opposed to turbulent) flow (Streeter [31]).)

\subsection{Image Acquisition}

The basic experimental rig is illustrated in Fig. 1. Experiments involved image sequences of fluid emulsions driven through a variety of physical devices. The emulsions consisted of a contrast medium mixed with a fluid and thereby yielded a spatially varying image intensity pattern. After emulsification, the contrast medium was dispersed in the fluid as tiny droplets $(1-20 \mu \mathrm{m})$. By the choice of a contrast medium with the same density as the fluid (1-bromohexadecane in water) the droplets were made neutrally buoyant and followed

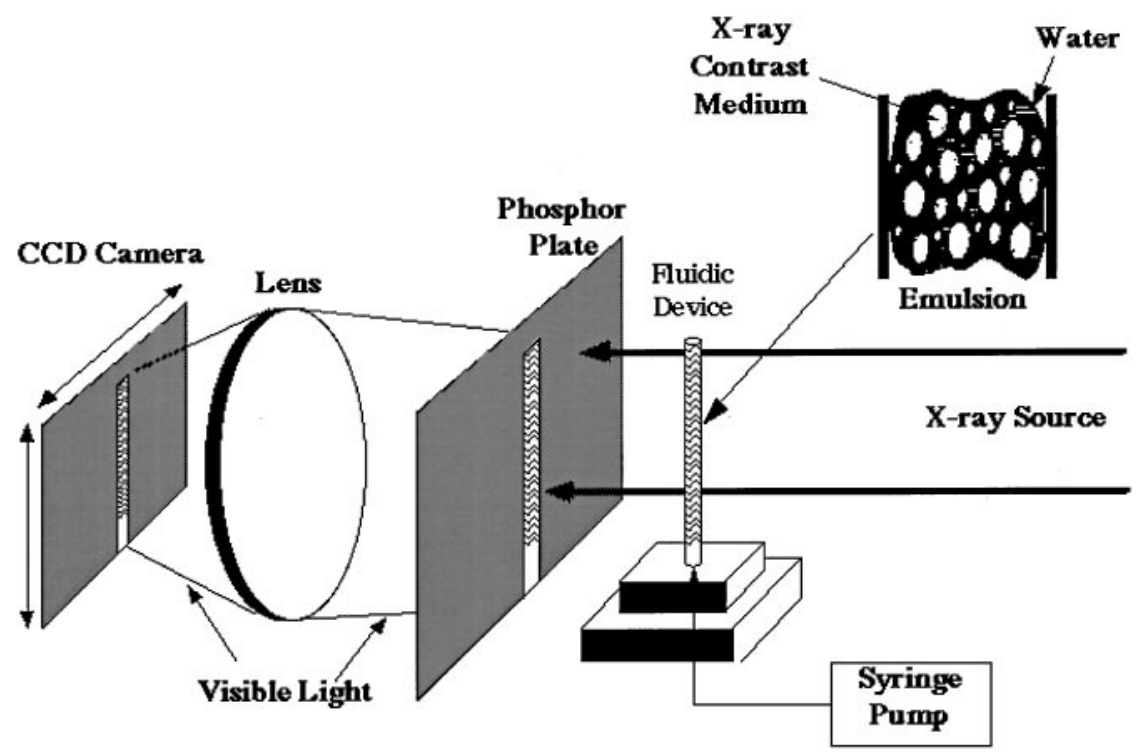

FIG. 1. Image acquisition. Image sequences of fluid emulsions driven through a variety of physical devices were used to evaluate the performance of the recovery algorithm. The flow was imposed by a mechanically driven syringe pump. Images were generated by a collimated monochromatic X-ray source in conjunction with a phosphor screen and optics to image onto a CCD imager for digitization. 
the imposed flow. The flow was generated by a mechanically driven syringe pump that could be controlled to force the fluid emulsion through the devices at 0.004 or $0.008 \mu \mathrm{l} / \mathrm{s}$. Images were generated by a collimated monochromatic X-ray source in conjunction with a phosphor screen and optics to image onto a CCD imager for digitization at 14-bit precision. This system is capable of operating with two sets of spatiotemporal resolution parameters. The first configuration has spatial resolution at $2.8 \mu \mathrm{m} /$ pixel with a temporal sampling rate of 0.4 frames/s and an exposure time of $500 \mathrm{~ms} /$ frame. The second configuration has spatial resolution a $1.6 \mu \mathrm{m} /$ pixel with a temporal sampling rate of $10 \mathrm{frames} / \mathrm{s}$ and an exposure time of $100 \mathrm{~ms} / \mathrm{frame}$.

\subsection{Synthetic Imagery}

Synthetic imagery allows the testing of computer vision algorithms in the face of known ground truth. Accordingly, synthetic images were generated to simulate the transmittance imagery that is acquired with the image acquisition rig of Section 3.1. The first simulated experimental preparation consisted of steady state fluid flow through cylindrical tubes. This preparation is of interest because the expected flow can be predicted analytically according to the fully developed circular pipe flow model [31]; therefore, recovered flows can be evaluated against physically meaningful model predictions. The pipe flow model dictates a parabolic displacement along the axis of the tube with the form

$$
\frac{v}{v_{\max }}=1-\frac{r^{2}}{R^{2}},
$$

where $R$ is the tube radius, $r$ is the perpendicular distance of any point in the tube from the central axis, $v_{\max }$ is the maximal displacement along the central axis, and $v$ is the displacement along the tube axis at point $r$. Flow in the orthogonal direction is taken as zero.

To mimic this setup, virtual balls were generated and randomly dispersed within a cylinder. The density and diameters of the balls were chosen to be in accord with the fluid emulsions used with the actual image acquisition rig. A raytracer was used to simulate the transmission of $\mathrm{X}$ rays through these structures according to a standard linear absorption model, as given by Eq. (12) in the Appendix. The simulated spatial resolution was $2.8 \mu \mathrm{m} /$ pixel. The gray-level resolution was 16 bits. A second image was raytraced after the spheres were shifted according to the pipe flow model (9). The left panel of Figure 2 shows an image from such a simulation experiment.

Simulated flow sequences were generated for tube diameters of 1000, 800, and $600 \mu \mathrm{m}$. For each tube, flow rates were simulated to yield a range of maximum image displacements. The flow-recovery algorithm was executed on the resulting image sequences. In these experiments $\lambda=0.01$, an empirically selected value. To quantify performance, the root mean square error between the recovered and simulated velocities in the direction of the tube axis was calculated in the following fashion,

$$
\operatorname{RMS}(\text { error })=\sqrt{\frac{\sum_{r=-D / 2}^{r=D / 2}\left(v_{\text {recovered }}(r)-v_{\text {simulated }}(r)\right)^{2}}{D}},
$$

with $D$ being the tube diameter. (The recovered velocities in the orthogonal direction were inconsequential.) The results are shown in the right Fig. 2. For small displacements the error 

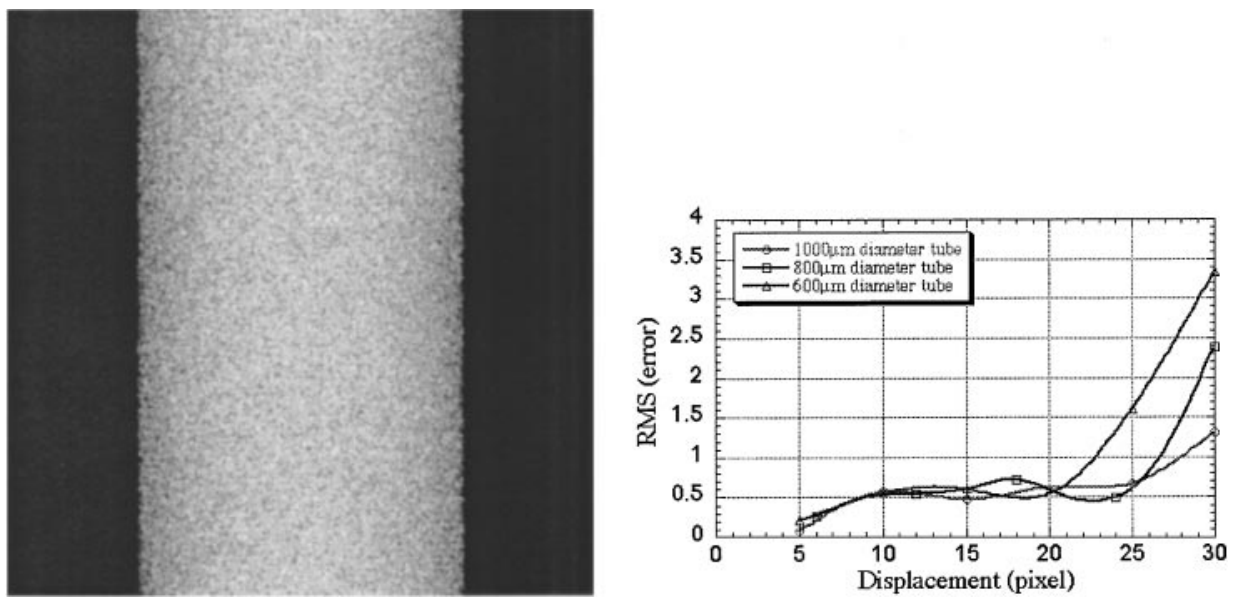

FIG. 2. Root mean square (RMS) error results using synthetic imagery: (left) a synthetic radiograph of emulsion particles flowing through a cylindrical tube; (right) the RMS error of recovered vs veridical displacements for a range of simulated tube diameters.

is small for all tubes. With increased displacement the error rises, especially for smaller tubes. While not apparent in these plots, the error typically comes as an underestimate of the true flow. The errors are due to the fact that the algorithm requires more spatial support in the recovery of larger displacements. The narrower tubes may not offer sufficient information to support the recovery of large displacements. Still, the algorithm proves capable of subpixel precision for an interesting range of experimental parameters.

The convergence of the flow-recovery algorithm is illustrated in Fig. 3. The RMS error is plotted as a function of iteration for two of the simulated tube flows: the lowest flow rate in the widest tube and the highest flow rate in the narrowest tube. These examples have been selected as they capture the extremes of the simulations (i.e., the smallest displacement with the most spatial support and the largest displacement with the least spatial support). In both cases the error decreases at a reasonable rate.

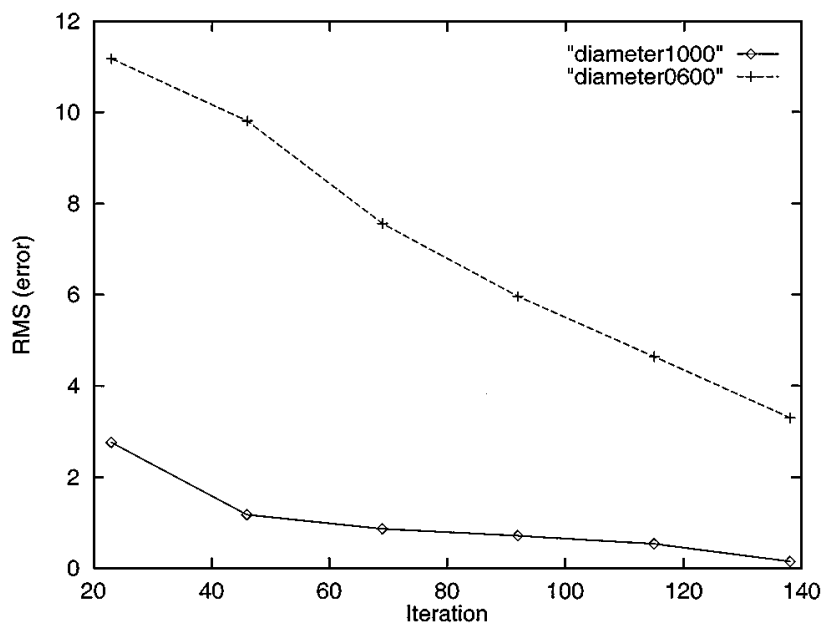

FIG. 3. Algorithm convergence as a function of the iteration number. The plots show RMS error in recovered displacement as a function of the iteration number for synthetic flow through two cylindrical tubes. 

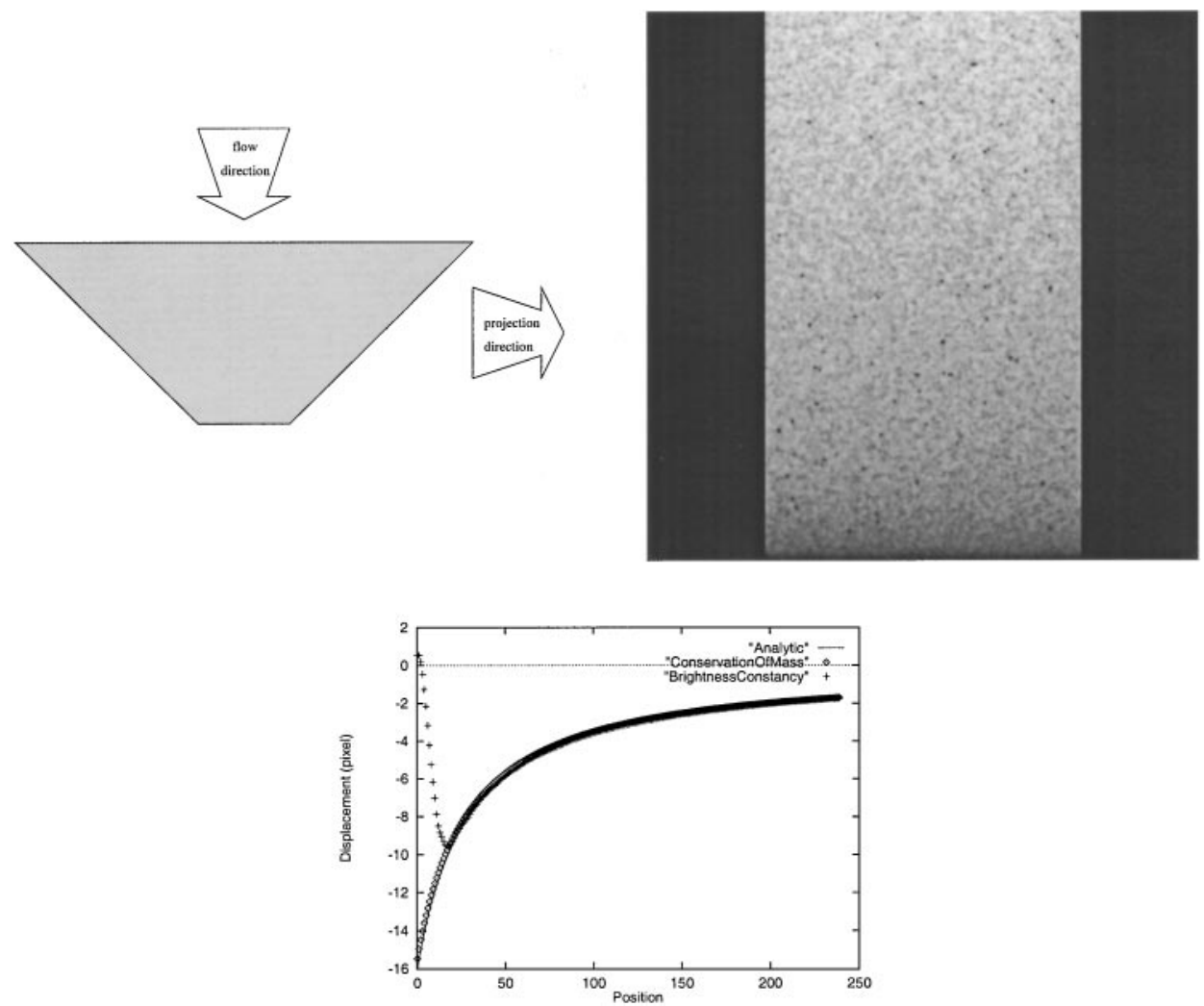

FIG. 4. Velocity profiles recovered from synthetic imagery of fluid flow through a converging channel. (upper left) A schematic of the simulated flow experiment. Flow is downward between a pair of converging plates open at the top and bottom (shown as a truncated triangle for simplicity; i.e., the plates extend from the plane of the figure) and projected along the horizontal axis. (upper right) A corresponding synthetic radiograph of emulsion particles flowing through the channel. The direction of projection yields a rectangular image region. (lower) The average recovered vertical flow profiles for conservation of mass, $\diamond$, and brightness constancy, + , constrained flow recovery algorithms. The analytically predicted flow profile for this experiment also is shown (solid line).

The second simulated experimental preparation consists of fluid flow between a pair of converging plates. A schematic of this device is shown in the upper left panel of Fig. 4. This preparation is of interest because the expected flow is well understood from an analytic point of view [31], with velocity increasing as the channel converges while trailing to zero along the channel walls. For present purposes, it is illustrative to take the transmittance projection of this three-dimensional flow in the direction that is shown in the schematic, i.e., along the horizontal. (A practical application of such a projection comes about when the optical depth of the device is too great to allow projection in the direction orthogonal to the schematic.) A simulated image for this preparation is shown in the upper right panel of Fig. 4. Notice that the average local intensity decreases from top to bottom as the projected matter decreases and that the convergent shape is no longer apparent as it is integrated across. For the simulated convergence angle, $\pi / 2$, the image displacement parallel to the direction of convergence has the form

$$
\frac{v}{v_{\max }}=-\frac{1}{y}
$$


where increasing $y$ is taken opposite to the direction of convergence with origin at the apex of convergence and $v_{\max }$ is the maximal displacement at the outlet, located at some positive $y$. Flow in the orthogonal direction is assumed to be zero. With reference to Fig. 4 the flow is downward and increasing from top to bottom. The results of executing the conservation of mass constrainted flow recovery algorithm, with $\lambda=0.01$, on a corresponding image pair is illustrated in the lower panel of Fig. 4. Here, the average recovered velocity in the vertical direction is plotted against image position. (Recovered velocity in the orthogonal direction was inconsequential.) For comparison, the analytically predicted velocity is shown in the same plot. The RMS error of the recovered flow is 0.2 pixel, mostly due to a slight tendency to underestimate the larger displacements. The plot also shows the results of replacing the conservation of mass constraint in the algorithm with the brightness constancy constraint (2), while keeping all other parameters constant. In this case, the recovered flow grossly underestimates the larger displacements. Correspondingly, the overall RMS error is elevated to 3.1 pixels.

\subsection{Natural Imagery}

Following on the experiments with synthetic imagery, the algorithm has been evaluated in the face of natural imagery of steady state flow through cylindrical tubes. In particular, the image acquisition rig described in Section 3.1 was used to capture the flow of fluid through small-diameter quartz capillary tubes. An initial set of experiments considered flow through an 800- $\mu \mathrm{m}$-diameter tube. Two flow rates were considered: 0.004 and $0.008 \mu \mathrm{l} / \mathrm{s}$. The spatial resolution was $2.8 \mu \mathrm{m} /$ pixel with a temporal sampling rate of $0.4 \mathrm{frame} / \mathrm{s}$ and an exposure time of $500 \mathrm{~ms} /$ frame. A single frame from the lower flow rate sequence is shown in Fig. $5 \mathrm{a}$.

The flow-recovery algorithm was executed on both the $0.004-$ and $0.008-\mu 1 / \mathrm{s}$ capillary tube image sequences. In these experiments, $\lambda=0.0001$, an empirically selected value. The recovered flow for the lower flow rate is shown as a vector plot in Fig. 5b. Qualitatively, the recovered flow is in agreement with inspection of the image sequence by eye: The
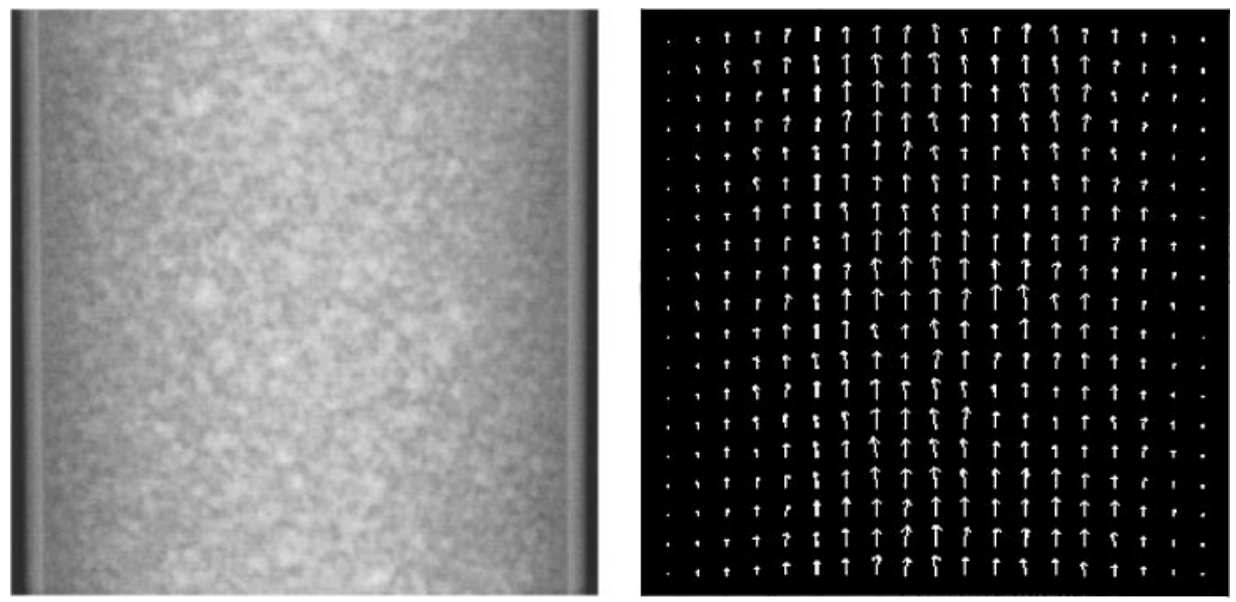

FIG.5. Velocity field recovered from natural imagery of fluid flow through a capillary tube. (left) A frame from a radiographic image sequence of an emulsion flowing through an 800 - $\mu$ m-diameter capillary tube. (right) The recovered velocity field from two successive frames. The recovered flow is in accord with one's visual impression upon viewing the image sequence. 

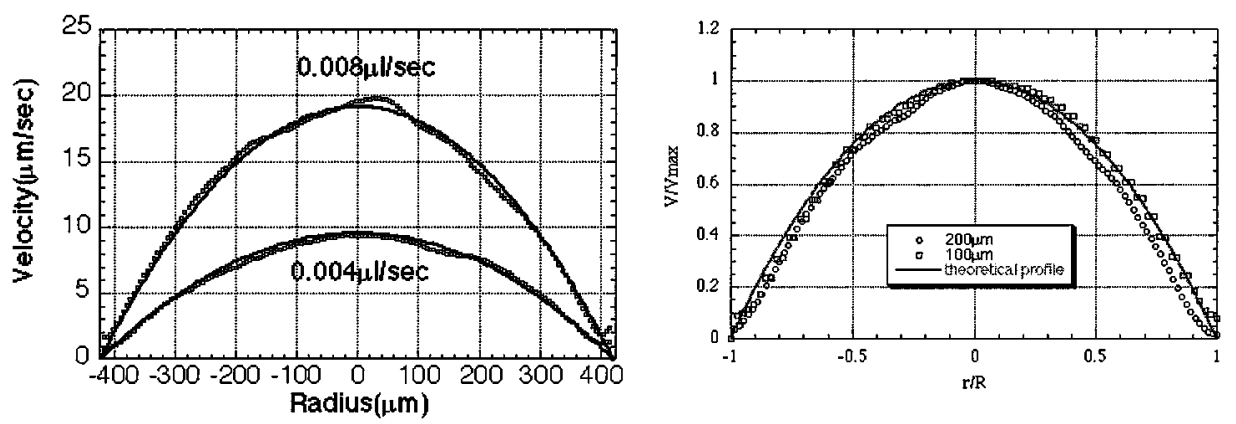

FIG. 6. Velocity profiles recovered from natural imagery of fluid flow through capillary tubes. (left) The average recovered axial flow profiles for two different flow rates through an 800 - $\mu \mathrm{m}$-diameter capillary tube (dotted lines). Analytically predicted flow profiles for these experiments also are shown (solid lines). (right) The recovered axial flow profiles for two different capillary tube diameters in dimensionless coordinates. The results for 200- and 100- $\mu$ m-diameter tubes are plotted with the symbols $\bigcirc$ and $\square$, respectively. The corresponding analytically predicted flow profile is shown as a solid line.

magnitude is greatest along the center line and trails toward zero at the side; the direction is predominantly along the tube axis (i.e., vertical), with somewhat more variation in the center than at the side. The average recovered velocity profiles in the axial direction are shown in Fig. 6a. For comparison, the profiles predicted by fully developed pipe flow (9) averaged along imaging rays also are plotted in Fig. 6. (Recall that the imaged flow should be an average of the three-dimensional flow due to the properties of transmittance imaging.) The recovered flow is in good agreement with the predictions of the model for both flow rates.

Additional capillary tube experiments have been executed for flow at $0.004 \mu \mathrm{l} / \mathrm{s}$ in capillary tubes with diameters of 200 and $100 \mu \mathrm{m}$. For these cases the spatial resolution was $1.6 \mu \mathrm{m} / \mathrm{pixel}$ with a temporal sampling of $10 \mathrm{frames} / \mathrm{s}$ and an exposure time of $100 \mathrm{~ms} / \mathrm{frame}$; $\lambda=0.00001$, an empirically selected value. The results for these experiments are collapsed into a single nondimensional plot in Fig. $6 \mathrm{~b}$. The radius of the tube, $R$, and the maximum velocity, $v_{\max }$, were used to nondimensionalize the plot. A pipe flow velocity profile (9) also is superimposed on this plot. In both cases, the recovered profiles are in reasonable agreement with the theoretical prediction. Interestingly, it appears that no previous study has documented the flow of fluids through cylindrical tubes with diameters under $1000 \mu \mathrm{m}$.

A second set of studies involved microradiography of more complicated devices where analytic predictions of the flow were not available. The first device was a ramped step channel. The second device was a serpentine channel. Both devices were etched in silicon and covered with glass. Parameters for both studies were the same: Injected flows were $0.004 \mu \mathrm{l} / \mathrm{s}$; the spatial resolution was $1.6 \mu \mathrm{m} / \mathrm{pixel}$ with a temporal sampling $10 \mathrm{frames} / \mathrm{s}$ and an exposure time of $100 \mathrm{~ms} /$ frame. $\lambda=0.00001$, an empirically selected value. An image of the ramped step channel and a recovered velocity field are shown in Fig. 7 . The results are in accord with one's visual impression. The flow is fastest at the upper inlet and loses speed while expanding thereafter; the speed is smallest near the channel walls. An image of the serpentine channel and a recovered velocity field are shown in Fig. 8. Again, the results are in accord with one's visual impression. The flow follows the channel's bends most closely along the boundaries and less so in the center; the speed is smallest near the channel walls. 


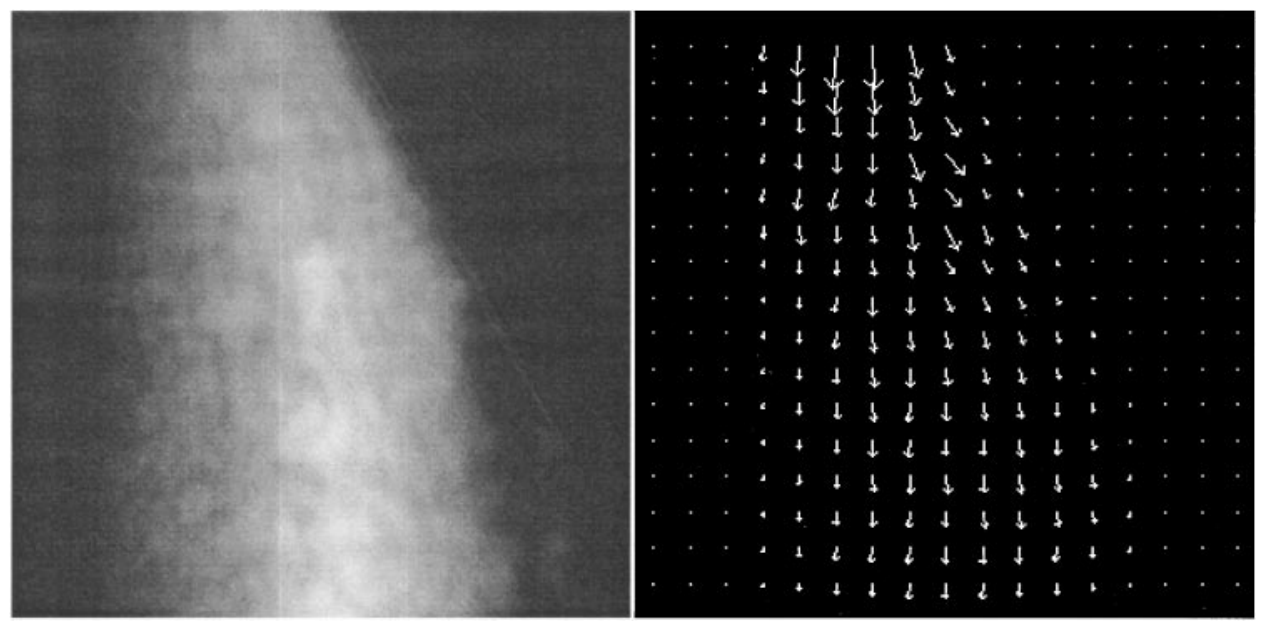

FIG. 7. Velocity field recovered from natural imagery of fluid flow through a ramped step channel. (left) A frame from a radiograph sequence of an emulsion flowing through a ramped step channel etched in silicon and covered with glass. (right) The velocity field recovered from two successive frames. The recovered flow is in accord with one's visual impression upon viewing the image sequence.

\subsection{Discussion}

In general, the algorithm for recovering fluid flow from image sequence data has performed well in empirical evaluation: For cases where the expected flow can be predicted analytically, the recoverd flow is in accord with theory. For complex flows, where the results cannot be predicted analytically, the recovered velocity fields agree with qualitative expectations. Also, the algorithm exhibits a reasonable rate of convergence.

In order to achieve the reported level of performance, it has been necessary to use more than one numerical value for the smoothing parameter, $\lambda$. Significantly, however, it has been

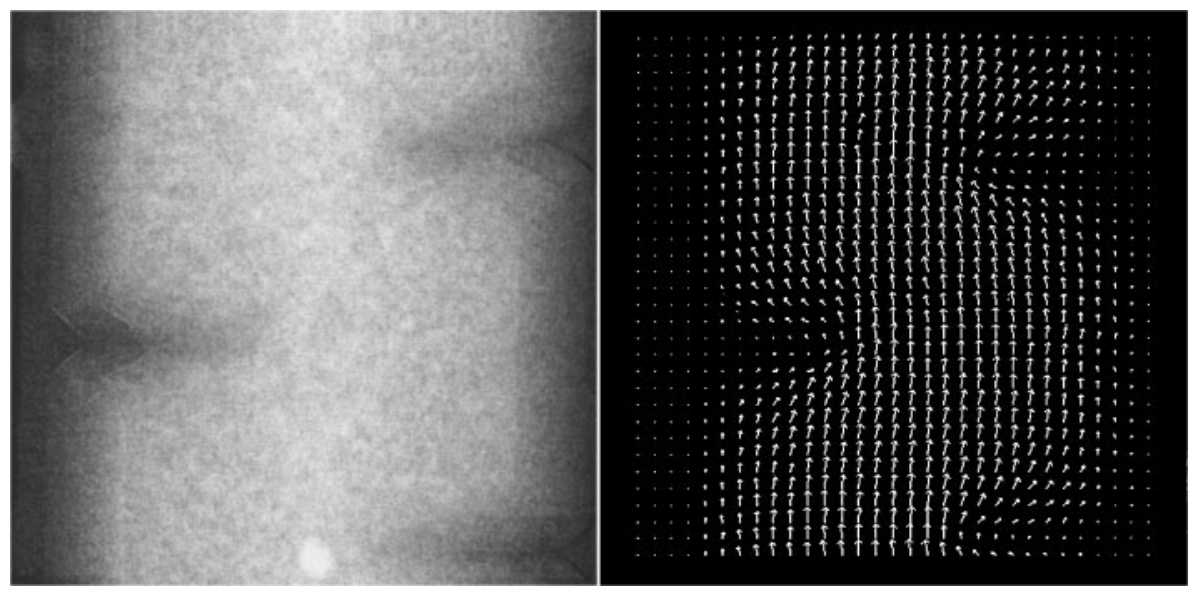

FIG. 8. Velocity field recovered from natural imagery of fluid flow through a serpentine channel. (left) A frame from a radiograph sequence of an emulsion flowing through a serpentine channel etched in silicon and covered with glass. (right) The velocity field recovered from two successive frames. The recovered flow is in accord with one's visual impression upon viewing the image sequence. 
possible to use a single value for each experimental configuration: 0.01 for the synthetic image experiments and 0.0001 and 0.00001 for the low and high (respectively) spatiotemporal resolution natural image experiments. More generally, the algorithm is reasonably stable with respect to minor variations in the value of $\lambda$ for any of the three configurations. For example, halving or doubling the value of $\lambda$ keeps the RMS error at the same order of magnitude. Further, the fact that it has been possible to use a single level of smoothing for multiple experiments with each configuration attests to this stability. Still, the level of smoothness is relatively large and can be seen as in conflict with previous results suggesting that recovered flow can lose accuracy due to oversmoothing (e.g., Song and Leahy [19]). This apparent discrepancy is likely due to the fact that the flows that are considered in the current paper are inherently smoother than those reported in the previous work. (For example, the flows studied by Song and Leahy included vortex structures that are not present in the current studies.) It also is worth noting that the level of smoothing that is reported in the current natural image studies is a reflection of the high level of noise that is inherent in the applied imaging methodology. Significantly, even given the weight that is placed on the smoothing term (4), the proposed algorithm does not reduce merely to interpolating the boundary conditions with first-order smoothness vector splines. The patterns of recovered flow away from the boundaries would not be present if that were the case. For illustration, consider the pipe flow examples. The "null normal flow" boundary condition (7) yields the same constraint on the left and right sides of the imaged channel. The natural boundary condition at the imaged channel ends, i.e., top and bottom as depicted in the figures, does not in and of itself vary across the horizontal dimension. Taking the preceding two observations in tandem, it becomes clear that the interpolation of the boundary conditions with first-order smoothing splines cannot yield variation of the flow profile as one proceeds along the horizontal dimension. In contrast, the recovered flow profile has a pronounced parabolic profile parameterized along the horizontal dimension.

Given the range of experimental results that have been presented in the current paper, it is interesting to consider how alternatives to the applied algorithm might fare if given the same data. At one extreme, it is worth noting that for the cylindrical pipe and converging plate experiments the flow is essentially one-dimensional. Correspondingly, one of the velocity components in the continuity equation (1) can be set to zero and the remaining component can be solved for uniquely given appropriate boundary conditions (although, in practice, some enforcement of smoothing (5) will still be necessary to combat the effects of noise). These observations suggest the applicability of a restricted one-dimensional version of the flow-recovery algorithm; however, the use of such an algorithm will necessarily be limited to situations where the considered flow is itself one-dimensional. It also is interesting to consider the applicability of previously developed algorithms that have made use of conservation of mass as bolstered by irrotational (Fitzpatrick [16], Fitzpatrick and Pedersen [17]) and small-divergence (Song and Leahy [19]) constraints. In this regard, it is illustrative to consider the two flow patterns that were given analytically, projected pipe flow (9) and projected converging plates (10). For the pipe flow case, notice that the curl of the flow, $\nabla \times \mathbf{v}$, evaluates to $-2 v_{\max } \frac{r}{R^{2}}$. Typically, this formula will evaluate to nonzero values and thereby calls into question use of the irrotational constraint for this case. This effect will be most pronounced when the magnitude of $r$ is relatively large (i.e., near the channel walls). For the converging plates case, notice that the divergence of the flow, $\nabla \cdot \mathbf{v}$, evaluates to $v_{\max } \frac{1}{y^{2}}$. Again, this formula generally evaluates to nonzero values and thereby calls into question use of the small divergence constraint for this case. 
Here, the effect will be most pronounced when the magnitude of $y$ is relatively small (i.e., near the channel outlet). Finally, it is appropriate to discuss the relative performance of conservation of mass and brightness constancy constrained algorithms, as illustrated by the converging plates preparation. Empirically, the brightness constancy algorithm exhibits a notably greater departure from ground truth than the conservation of mass algorithm. The inferior performance arises as the brightness constancy algorithm attempts to establish correspondence between similar image brightness patterns where it is not appropriate to do so. In particular, recall that for projected converging plates, the flow pattern decreases in its local average gray level along its path even while increasing in speed due to the convergent geometry and direction of projection. In contrast, the conservation of mass constraint accurately captures this state of affairs.

\section{SUMMARY}

An algorithm for measuring fluid flow from image sequences has been presented. The algorithm was derived from three constraints. First, a physics-based flow continuity equation, motivated by the principle of conservation of mass, was used to relate image data to fluid behavior. Second, a generic smoothness constraint was imposed to regularize the image data. Third, realistic boundary conditions were used to further ensure the physical significance of the recovered flow. The calculus of variations was used to combine the constraints to yield a pair of partial differential equations that relate the spatiotemporal derivatives of image intensity to the underlying flow components. These equations were discretized to produce an iterative solution for flow given image sequence data. A corresponding numerical algorithm has been implemented in the $\mathrm{C}$ programming language for execution on standard computer workstations. This instantiation has received preliminary testing on both synthetic and natural image fluid flows. The results of these experiments show the promise of the approach as a measurement technique for fluid mechanics. It is conjectured that the algorithm also could be applicable to other imaged flows that are governed by similar physical constraints. More generally, this research provides an illustration of the place of physical analysis in the derivation of effective computer vision algorithms.

\section{APPENDIX: TRANSMITTANCE IMAGE FORMATION}

Conservation of mass is a reasonable constraint for fluid flow. However, it is less obvious that the flow captured in a corresponding image sequence is subject to such a constraint. This Appendix shows that for transmittance imagery, the constraint is indeed appropriate. In particular, it is shown that the two-dimensional transmittance image of a three-dimensional flow that respects three-dimensional conservation of mass is a two-dimensional flow that respects two-dimensional conservation of mass, subject to certain constraints on normal flow. The results that are derived in this Appendix are an extension of previous work on the analysis of transmittance imaging (Fitzpatrick [18]). The current derivation adds to previous results in its analysis of the relevant boundary conditions. In particular, previous results required that the boundaries of integration be outside the region for which the flow is nonzero or that the flow at opposite boundary points along the path of integration be equal, whereas the current analysis shows that it suffices for the normal flow along the boundaries of integration to be equal to zero. Further, the relationship between boundary conditions in the world and boundary conditions in the image is made explicit. 
Let $\rho(x, y, z, t)$ be the density of a fluid, a function of spatial coordinates, $(x, y, z)$, and time, $t$, that is subject to a velocity field $\mathbf{V}(x, y, z, t)=(U(x, y, z, t), V(x, y, z, t)$, $W(x, y, z, t))$. Assuming that this fluid respects the conservation of mass, the density and velocity are related according to

$$
\nabla \cdot(\rho \mathbf{V})+\frac{\partial \rho}{\partial t}=0
$$

with $\nabla$ the three-dimensional spatial gradient operator. Transmittance image formation can be modeled as yielding intensities that are proportional to an object's density, integrated along the path of the impinging energy. For incident energy parallel to the $z$ direction and an image recorded in the $(x, y)$ plane, the image is given as

$$
E(x, y, t)=\int_{z_{1}(x, y)}^{z_{2}(x, y)} \rho(x, y, z, t) d z
$$

where $z_{1}(x, y)$ and $z_{2}(x, y)$ are the bounding surfaces of the specimen that is being imaged. (Here, the mass absorption coefficient has been absorbed into the density for the sake of convenience.) This formulation is a reasonable model of collimated monochromatic X-ray image formation [38], e.g., as used in the experimental work described in this paper. (Key assumptions underlying this model of image formation include monochromatic photons, detector robustness to scatter, narrow beam, and small aperture. These constraints are reasonably well satisfied in the experimental apparatus used for the empirical results reported in the current paper. For example, this apparatus employs a highly collimated synchroton $\mathrm{X}$-ray source with spatial resolution approaching $1 \mu \mathrm{m}$ and energies, $e$, precisely tuned with narrow bandpass $\frac{\delta e}{e} \approx 6 \times 10^{-4}$ [1]. More commonplace devices, e.g., medical CT devices, will have images less well modeled by the idealized relationship (12). For example, the X-ray beam in such devices contains a broader range of energies that attenuate by different amounts. Nevertheless, the idealized imaging relationship can still be used with practical advantage, as attested to by its providing the basis for tomographic reconstruction [38].) Application of this model of image formation to the continuity equation (11) suggests integration along the $z$ axis according to

$$
\int_{z_{1}(x, y)}^{z_{2}(x, y)} \nabla \cdot(\rho \mathbf{V}) d z+\int_{z_{1}(x, y)}^{z_{2}(x, y)} \frac{\partial \rho}{\partial t} d z=0 .
$$

The first term (i.e., the spatial term) of the integrated continuity equation (13) can be rewritten as

$$
\int_{z_{1}}^{z_{2}} \nabla \cdot(\rho \mathbf{V}) d z=\int_{z_{1}}^{z_{2}} \nabla x, y \cdot\left(\rho \mathbf{V}_{x, y}\right) d z+\int_{z_{1}}^{z_{2}} \frac{\partial}{\partial z}(\rho W) d z,
$$

where $\mathbf{V}_{x, y}(x, y, z, t)=(U(x, y, z, t), V(x, y, z, t))$ and $\nabla x, y$ is the two-dimensional gradient operator in $(x, y)$. It is useful to bring the (two-dimensional) gradient operator to the outside of the first term on the right-hand side of the expanded integral (14). To perform this operation, recall that, in general, if

$$
F(r)=\int_{\alpha(r)}^{\beta(r)} f(r, s) d s
$$


then

$$
F^{\prime}(r)=\beta^{\prime}(r) f(r, \beta(r))-\alpha^{\prime}(r) f(r, \alpha(r))+\int_{\alpha}^{\beta} \frac{\partial f(r, s)}{\partial r} d s
$$

(Buck [39]). Applying this formula to the first integral on the right-hand side of (14) yields

$$
\int_{z_{1}(x, y)}^{z_{2}(x, y)} \nabla_{x, y} \cdot\left(\rho \mathbf{V}_{x, y}\right) d z=\nabla_{x, y} \cdot \int_{z_{1}}^{z_{2}} \rho \mathbf{V}_{x, y} d z-\left[\left(\nabla_{x, y} z\right) \cdot\left(\rho \mathbf{V}_{x, y}\right)\right]_{z_{1}}^{z_{2}}
$$

The second integral on the right-hand side of (14) evaluates to $[\rho W]_{z_{1}}^{z_{2}}$. This expression can be recombined with the rightmost term of (15) to yield

$$
\left[-(\nabla x, y z) \cdot\left(\rho \mathbf{V}_{x, y}\right)\right]_{z_{1}}^{z_{2}}+[\rho W]_{z_{1}}^{z_{2}}=[\rho \mathbf{n} \cdot \mathbf{V}]_{z_{1}}^{z_{2}}
$$

with $\mathbf{n}=\left(-\frac{\partial z}{\partial x},-\frac{\partial z}{\partial y}, 1\right)$ normal vectors to the surfaces $z_{1}$ and $z_{2}$. The right-hand side of this expression can be physically interpreted as material flow in the normal direction weighted by the surface gradient, evaluated at the extremes of the specimen. Equation (14) now can be written as

$$
\int_{z_{1}}^{z_{2}} \nabla \cdot(\rho \mathbf{V}) d z=\nabla x, y \cdot \int_{z_{1}}^{z_{2}} \rho \mathbf{V}_{x, y} d z+[\rho \mathbf{n} \cdot \mathbf{V}]_{z_{1}}^{z_{2}}
$$

This new form of the spatial part of the integrated continuity equation (16) can be substituted into the original integrated continuity equation (13) to yield

$$
\nabla x, y \cdot \int_{z_{1}}^{z_{2}} \rho \mathbf{V}_{x, y} d z+\int_{z_{1}}^{z_{2}} \frac{\partial \rho}{\partial t} d z+[\rho \mathbf{n} \cdot \mathbf{V}]_{z_{1}}^{z_{2}}=0
$$

Now, define a new two-dimensional velocity field as the density-weighted average of the original three-dimensional velocity field; i.e., let

$$
\mathbf{v} \equiv \frac{\int_{z_{1}}^{z_{2}} \rho \mathbf{V}_{x, y} d z}{\int_{z_{1}}^{z_{2}} \rho d z}
$$

Using this two-dimensional velocity (18) along with the model of image formation (12) allows the integrated continuity equation to be interestingly rewritten as

$$
\nabla x, y \cdot E \mathbf{v}+\frac{\partial}{\partial t} E=-[\rho \mathbf{n} \cdot \mathbf{V}]_{z_{1}}^{z_{2}}
$$

For the case of null normal flow at the boundaries, the right-hand side of this last equation vanishes. In this case the transmittance image of a three-dimensional flow that observes continuity is a two-dimensional flow that observes continuity, with the flow being the density-weighted average of the three-dimensional flow, i.e., definition (18). For example, this is the situation in the experiments that are reported in this paper. In contrast to the case of null normal flow at the boundaries, the general projected flow expression violates twodimensional continuity. Equation (19) shows that this deviation from continuity is governed by the material flow across the boundaries of the projected specimen. 
Finally, it is of interest to understand the image ramifications of the three-dimensional world null normal flow boundary condition

$$
[\mathbf{N} \cdot \mathbf{V}]_{\partial \Omega}=0,
$$

where $\partial \Omega$ is the three-dimensional boundary of a specimen of interest. In order for a part of the three-dimensional boundary $\partial \Omega$ to appear as a boundary, $\partial \omega$, in the image it must be positioned relative to the sensor as an "occluding contour." Keeping the geometry of image formation introduced above (collimated rays along the $z$ axis impinging on the $(x, y)$-plane image), this condition can be captured as

$$
\mathbf{N} \cdot(0,0,1)=0,
$$

implying that along such contours, $\mathbf{N}=\left(N_{x}, N_{y}, 0\right)$. When the constraint that the $z$ component of $\mathbf{N}$ is equal to 0 is placed on the three-dimensional null normal flow condition (20), it yields

$$
\left(N_{x}, N_{y}\right) \cdot\left(V_{x}, V_{y}\right)=0 .
$$

Following the operative geometry of image formation, let $\mathbf{n} \equiv\left(N_{x}, N_{y}\right)$ be the (image) normal along imaged boundary contours, $\partial \omega$. Combining this definition of $\mathbf{n}$ and the definition of $\mathbf{v}$ (18) with the constraint (21) shows that that the three-dimensional null normal flow boundary condition (20) implies a two-dimensional image domain null boundary condition,

$$
[\mathbf{n} \cdot \mathbf{v}]_{\partial \omega}=0
$$

as presented earlier in this paper as Relationship (7).

\section{ACKNOWLEDGMENTS}

J. Dunsmuir provided assistance in the acquisition of the microradiographs. J. R. Bergen, M. H. Brill, B. Huber, S. Peleg, R. Samtaney, and C. D. Spence provided valuable comments on this work. The anonymous reviewers also provided useful commentary.

\section{REFERENCES}

1. A. M. Lanzillotto, T. S. Leu, M. J. Amabile, and R. P. Wildes, An investigation of microstructure and microdynamics of fluid flow in MEMS, in Proceedings of the American Society of Mechanical Engineers Conference, 1996, pp. 789-796.

2. A. Amini, A scalar function formulation for optical flow, in Proceedings of the European Conference on Computer Vision, 1994, pp. 125-131.

3. H. Nogawa, Y. Nakajima, Y. Sato, and S. Tamura, Acquisition of symbolic description from flow fields: A new approach based on a fluid model, IEEE Trans. Pattern Anal. Mach. Intell. 19(1), 1997, 58-63.

4. I. Cohen and I. Herlin, Non uniform multiresolution method for optical flow and phase portrait models: Environmental applications, Internat. J. Comput. Vision 33(1), 1999, 29-49.

5. R. Larsen, K. Conradsen, and B. K. Ersbøll, Estimation of dense image flow fields in fluids, IEEE Trans. Geosci. Remote Sensing 36(1), 1998, 256-264.

6. E. Memin and P. Perez, Fluid recovery by coupling dense and parametric vector fields, in Proceedings of the IEEE International Conference on Computer Vision, 1999, pp. 620-625. 
7. S. Webb, The Physics of Medical Imaging, Institute of Physics Publishing Limited, London, 1988.

8. A. Del Bimbo, P. Nesi, and J. L. C. Sanz, Analysis of optical flow constraints, IEEE Trans. Image Process. 4(4), 1995, 460-469.

9. J. K. Aggarwal and N. Nandhakumar, On the computation of motion from image sequences-A review, Proc. IEEE 76(8), 1988, 917-935.

10. S. S. Beauchemin and J. L. Barron, The computation of optical flow, ACM Comput. Surv. 27(3), 1995, $433-467$.

11. B. K. P. Horn, Robot Vision, MIT Press, Cambridge, MA, 1986.

12. N. Cornelius and T. Kanade, Adapting optical-flow to measure object motion in reflectance and x-ray image sequences, in Proceedings of the ACM Workshop on Motion, 1983, pp. 50-58.

13. S. Negahdaripour and C. H. Yu, A generalized brightness change model for computing optical flow, in Proceedings of the IEEE International Conference on Computer Vision, 1993, pp. 2-11.

14. H. H. Nagel, On a constraint equation for the estimation of displacement rates in image sequences, IEEE Trans. Pattern Anal. Mach. Intell. 11(1), 1989, 13-30.

15. B. G. Schunk, The motion constraint equation for optical flow, in Proceedings of the IAPR International Conference on Pattern Recognition, 1984, pp. 20-22.

16. J. M. Fitzpatrick, A method for calculating velocity in time dependent images based on the continuity equation, in Proceedings of the IEEE Conference on Computer Vision and Pattern Recognition, 1985, pp. 78-81.

17. J. M. Fitzpatrick and C. A. Pedersen, A method for calculating fluid flow in time dependent density images, Electron. Imag. 1, 1988, 347-352.

18. J. M. Fitzpatrick, The existence of geometrical density-image transformations corresponding to object motion, Comput. Vision Graphics Image Process. 44, 1988, 155-174.

19. S. M. Song and R. M. Leahy, Computation of 3-D velocity fields from 3-D cine and ct images of a human heart, IEEE Trans. Medical Imaging 10(3), 1991, 295-306.

20. A. Del Bimbo, P. Nesi, and J. L. C. Sanz, Optical flow estimation by using classical and extended constraints, in Proceedings of the International Workshop on Time-Varying Image Processing and Moving Object Recognition, 1993, pp. 351-358.

21. B. Jähne and S. Waas, Optical wave measurement technique for small scale water surface waves, in Proceedings of Advances in Optical Instruments for Remote Sensing, 1989, pp. 147-152.

22. H. G. Maas, A. Stefanidis, and A. Gruen, Feature tracking in 3-D fluid tomography sequences, in Proceedings of the IEEE Conference in Image Processing, 1994, pp. 530-534.

23. R. J. Adrian, Particle imaging techniques for experimental fluid mechanics, Annual Rev. Fluid Mech. 23, 1991, 261-304.

24. L. Lourenco, A. Kromapalli, and C. A. Smith, Particle image velocimetry, in Advances in Fluid Mechanics Measurements (M. Gadel-Hak, Ed.), Springer-Verlag, Berlin, 1989.

25. C. E. Willert and M. Gharib, Digital particle image velocimetry, Experiments in Fluids 10, 1991, 181-193.

26. J. Zhong, T. S. Huang, and R. J. Adrian, Extracting 3D vortices in turbulent fluid flow, IEEE Trans. Pattern Anal. Mach. Intell. 20(2), 1998, 193-199.

27. R. M. Ford, R. N. Strickland, and B. A. Thomas, Image models for 2-D flow visualization and compression, CVGIP: Graphical Models Image Process. 56(1), 1994, 75-93.

28. R. M. Ford and R. N. Strickland, Representing and visualizing fluid flow images and velocimetry data by nonlinear dynamical systems, Graphical Models Image Process. 57(6), 1995, 462-482.

29. R. P. Wildes, M. J. Amabile, A. M. Lanzillotto, and T. S. Leu, Experiments with an algorithm for recovering fluid flow from video imagery, in Proceedings of the DARPA Image Understanding Workshop, 1997, pp. 185-192.

30. R. P. Wildes, M. J. Amabile, A. M. Lanzillotto, and T. S. Leu, Physically based fluid flow recovery from image sequences, in Proceedings of the IEEE Conference on Computer Vision and Pattern Recognition, 1997, pp. 969-975.

31. V. L. Streeter, Handbook of Fluid Dynamics, McGraw-Hill, New York, 1961.

32. A. N. Tikhonov and V. Y. Arsenin, Solutions of Ill-Posed Problems, Winston, Washington, DC, 1977.

33. B. K. P. Horn and B. G. Schunck, Determining optical flow, Artificial Intelligence 17(1-3), 1981, $185-203$. 
34. R. Courant and D. Hilbert, Methods of Mathematical Physics, Wiley, New York, 1953.

35. G. Dahlquist and A. Björck, Numerical Methods, Prentice Hall, Englewood Cliffs, NJ, 1974.

36. J. R. Bergen, P. Anandan, K. Hanna, and R. Hingorani, Hierarchical model-based motion estimation, in Proceedings of the European Conference on Computer Vision, 1991, pp. 5-10.

37. V. E. Cosslett, A. Engstrom, and H. H. Pattee, X-Ray Microscopy and Microradiography, Academic Press, New York, 1957.

38. A. Kak and M. Slaney, Principles of Computerized Tomographic Imaging, IEEE Comput. Soc. Press, New York, 1988.

39. R. C. Buck, Advanced Calculus, McGraw-Hill, New York, 1965.

Statement of ownership, management, and circulation required by the Act of October 23, 1962 , Section 4369 , Title 39. United States Code: of

\section{COMPUTER VISION AND IMAGE UNDERSTANDING}

Published monthly by Academic Press, 6277 Sea Harbor Drive, Orlando, FL 32887-4900. Number of issues published annualty: 12. Editor: Dr. Avinash C. Kak, Robot Vision Lab, 1285 EE Building. Purdue Universily, West Lafayette, IN 47907.

Owned by Academic Press, 525 B Street, Suite 1900, San Diego, CA 92101-4495. Known bondholders, mortgagees, and other security holders owning or holding 1 percent or more of total amount of bonds, mortgages, and other securities: None.

Paragraphs 2 and 3 include, in cases where the stockholder or security holder appears upon the books of the company as trustee or in any other fiduciary relation, the nante of the person or corporation for whom such trustee is acting. also the statements in the two paragraphs show the affiant's full knowledge and belief as to the circumstances and conditions under which stockholders and security holders who do not appear upon the books of the company as trustees, hold stock and securities in a capacity other than that of a bona fide owner. Names and addresses of individuals who are stockholders of a corporation which itself is a stockholder or holder of bonds, mortgages, or other securities of the publishing corporation have been included in paragraphs 2 and 3 when the interests of such individuals are equivalent to 1 percent or more of the total amount of the stock or securities of the publishing corporation.

Total no. copies printed: average no. copies each issue during preceding 12 months: 1826; single issue nearest 10 filing date: 1356. Paid circulation (a) to term subscribers by mail, carrier delivery, or by other means: average no. copies each issue during preceding 12 months: 456 ; single issue nearest to filing date: 343 . (b) Sales through agents news dealers, or otherwise: average no. copies each issue during preceding 12 months: 682 ; single issue nearest to filing date: 531. Free distribution (a) by mail: average no, copies each issue during preceding 12 months: 55 ; single issue nearest to filing date: 55 . (b) Outside the mail: average no. copies each issue during preceding 12 months: 20 ; single issue nearest to tiling date: 20 . Total no. of copies distributed: average no. copies each issue during preceding 12 months: 1213 ; single issue nearest to filing date: 949 . Percent paid and/or requested circulation; average percent each issue during preceding 12 months: $94 \%$; single issue nearest to filing date: $92 \%$. 